\title{
Hourglass Effects for Asymmetric Colliders *
}

\author{
Miguel A. Furman \\ Lawrence Berkeley Laboratory, Berkeley, CA 94720
}

\section{Abstract}

We give the expressions for the geometrical reduction factor of the lumincsity and the geometrical beam-beam "aggravating factor" for the general asymmetric case, for tri-gaussian bunches colliding head-on. With these formulas we attempt a (limited) analytic understanding of the multiparticle tracking simulations carried out for the proposed SLAC/LBL/LLNL B factory [1] when parasitic crossings are ignored. We conclude the following: (a) the geometrical reduction in luminosity is $\sim 6 \%$ relative to the zero-bunch-length (nominal) value; (b) only the vertical beam-beam parameter of the LER is significantly altered by the hourglass effect: the geometrical enhancement of the central positron's vertical beam-beam parameter is $\sim 10 \%$ relative to the nominal value, and (c) the positrons at the head or tail of the bunch have vertical beam-beam parameters much larger than nominal. We discuss the electromagnetic disruption effect only qualitatively. This effect probably compensates (or overcompensates) the geometrical reduction of the luminosity, and it is possibly detrimental for the beam-beam parameters. This article summarizes Ref. [2].

\section{INTRODUCTION}

Although proposed B factories [1] call for designs that are asymmetric in energy, beam current and emittances, they also invoke to a greater or lesser degree a "transparency condition" by virtue of which the beam sizes are pairwise equal [3]. Because of the beam-beam interaction, however, the beams become different in size at least to some degree. Expressions available in the literature $[4,5,6]$ for the hourglass factors for the luminosity and beam-beam parameters assume some sort of equality among the beam sizes or lattice functions. In this note we provide generalizations that are applicable to the most general asymmetric case, when the four beta-functions and the six rms beam sizes are arbitrary. With these formulas we attempt a (limited) analytic understanding of the multiparticle tracking resuits for the proposed SLAC/LBL/LLNL B factory [1] when parasitic collisions are ignored.

-Work supported by the Director, Office of Energy Research, Office of High Energy and Nuclear Phyoics, High Energy Phymics Division, of the U.S. Department of Energy under Contract Number DE-AC03-76SF00098.

\section{LUMINOSITY}

Consider two bunches moving in equal and opposite directions with speed $c$, with tri-gaussian particle distributions, such that the centers collide at the optical interaction point (IP, $s=0$ ) with no displacement. We assume that the interaction region is a dispersionless drift section and that the IP is a symmetry point of the lattice. Then the transverse rms sizes $\sigma_{x+1}, \sigma_{y+}, \sigma_{x-}$ and $\sigma_{y-}$ have an $s$ dependence of the form $\sigma^{2}=\sigma^{* 2} \times\left(1+s^{2} / \beta^{* 2}\right)$. The hourglass reduction factor for the luminosity is [2]

$$
R\left(t_{x}, t_{y}\right) \equiv \frac{\mathcal{L}}{\mathcal{L}_{0}}=\int_{-\infty}^{\infty} \frac{d t}{\sqrt{\pi}} \frac{\exp \left(-t^{2}\right)}{\sqrt{\left(1+t^{2} / t_{x}^{2}\right)\left(1+t^{2} / t_{y}^{2}\right)}}
$$

where $\mathcal{L}$ is the actual luminosity and $\mathcal{L}_{0}$ is the luminosit; in the zero-bunch-length limit,

$$
\mathcal{L}_{0}=\frac{f_{0} N_{+} N_{-}}{2 \pi \sqrt{\left(\sigma_{x+}^{* 2}+\sigma_{x-}^{* 2}\right)\left(\sigma_{y+}^{* 2}+\sigma_{y-}^{* 2}\right)}}
$$

where $f_{b}$ is the bunch collision frequency, and $t_{x}$ is defined by

$$
t_{x}^{2}=\frac{2\left(\sigma_{x+}^{* 2}+\sigma_{x-2}^{* 2}\right)}{\left(\sigma_{t+}^{2}+\sigma_{--}^{2}\right)\left(\sigma_{x+}^{* 2} / \beta_{x+}^{* 2}+\sigma_{x-}^{* 2} / \beta_{x-1}^{* 2}\right)}
$$

with a corresponding expression for $t_{y}$. The superscript * refers to the IP and $\sigma_{z \pm}$ are the rms bunch lengths. We exhibit $R\left(t_{x}, t_{y}\right)$ in Fig. 1.

It is easy to see from $E q$. (1) that $R$ is always $<1$, except that $R(\infty, \infty)=1$, as it should.

If both beams are flat, with $\sigma_{x \pm} \gg \sigma_{y \pm}$, then $t_{x} \gg 1$ and (1) can be expressed in terms of a modified Bessel function. This result is of similar form to that of Ivanov et al. [5] but is of moire general validity because it does not assume $\beta_{y_{+}}^{*}=\beta_{y_{-}}^{*}$ or $\sigma_{a_{+}}=\sigma_{s_{-}}$or $\sigma_{y_{+}}^{*}=\sigma_{y_{-}}^{*}$.

If the beams are such that $t_{x}=t_{y}$ (which may happen naturally in a round-beam design), then (1) can be expressed in terms of the complementary error function.

\section{Beam-Beam Parameters}

We focus on a single particle, say a positron, as it passes through the opposing electron bunch. In a first-order calculation we can assume that the particle follows a straight line trajectory with constant speed $c$. We assume that this particle is close to the collision axis and is displaced longitudinally by a finite distance $z$ from the center of its 


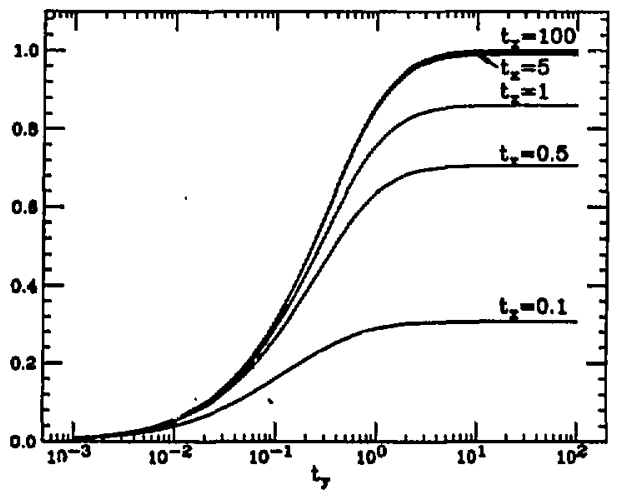

Figure 1: The luminosity reduction factor, Eq. (1), plotted vs. $t_{y}$ for various values of $t_{x}$.

own bunch. We assume that $z$ is constant during the collision process (or during several successive collisions), which is a very good approximation in practice. Then the "aggravating factor" [4] for the positron's vertical beam-beam parameter is [2],

$$
\begin{aligned}
& R_{y+}(z) \equiv \xi_{y+}(z) / \xi_{0 y+}= \\
& \int_{-\infty}^{\infty} \frac{d t}{\sqrt{\pi}} \frac{\left(1+t^{2} / t_{1}^{2}\right) \exp \left(-\left(t-t_{0}\right)^{2}\right)}{\sqrt{1+t^{2}} / t_{2}^{2}\left(v \sqrt{1+t^{2} / t_{2}^{2}+h \sqrt{1+t^{2} / t_{3}^{2}}}\right)}
\end{aligned}
$$

where $h=\sigma_{x_{-}}^{n_{-}} /\left(\sigma_{x_{-}}^{*}+\sigma_{y_{-}}^{*}\right), v=\sigma_{y_{-}}^{*} /\left(\sigma_{x_{-}}^{*}+\sigma_{y_{-}}^{*}\right)$, $t_{0}=z / \sqrt{2} \sigma_{,-}, t_{1}=\sqrt{2} \beta_{y_{+}}^{*} / \sigma_{,-}, t_{2}=\sqrt{2} \hat{\beta}_{y-}^{*} / \sigma_{,-}$and $t_{3}=\sqrt{2} \beta_{x-}^{*} / \sigma_{1} .$. . The nominal (zero-bunch-length) vertical beam-beam parameter $\xi_{0 y+}$ of the central positron is

$$
\xi_{0 y_{+}}=\frac{r_{0} N_{-} \beta_{y+}^{*}}{2 \pi \gamma_{+} \sigma_{y-}^{*}\left(\sigma_{x-}^{*}+\sigma_{y-}^{*}\right)}
$$

where $r_{0}$ is the classical electron radius and $\gamma_{+}$is the usual relativistic factor. The expressions for the remaining three beam-beam parameters $\xi_{x+}, \xi_{x-}$ and $\xi_{y-}$ are obtained from Eqs. (4) and (5) by the substitutions $x \mapsto y$ and/or +-- in $h, v$ and the $t_{i}$ 's.

It should be noted that the aggravating factors can be $>1$ or $<1$, as opposed to the luminosity reduction factor, which is always $<1$. However, if $\beta_{x+}^{*}=\beta_{y+}^{*}=\beta_{x-}^{*}=\beta_{y-}^{*}$ we obtain

$$
R_{x+}(z)=R_{y+}(z)=R_{z-}(z)=R_{y-}^{-}(z)=1
$$

for all $z$ regardless of the beam zizes. This allows, in principle, for the possibility of designing the lattice so that there is no hourglass effect on the beam-beam parameters.

If the beams are flat such that $\sigma_{x+} \gg \sigma_{y+}, \sigma_{x-} \gg \sigma_{y-}$, $\beta_{z+}^{*} \gg \beta_{y+}^{*}$ and $\beta_{x_{-}}^{*} \gg \beta_{y-}^{*}$, Eq. (4) yields, for the central particle, $R_{x_{+}}(0) \simeq R_{x_{-}}(0) \simeq 1$, and

$$
R_{y \dot{r}}(0) \simeq \frac{t_{2}}{2 \sqrt{\pi}} e^{t_{2}^{3} / 2}\left[(2-\rho) K_{0}\left(t_{2}^{2} / 2\right)+\rho K_{1}\left(t_{2}^{2} / 2\right)\right]
$$

where $\rho \equiv\left(t_{2} / t_{1}\right)^{2}$ and $K_{0}, K_{1}$ are Bessel functions. $R_{y-}(0)$ is obtained from (7) by exchanging $t_{1} \rightarrow t_{2}$.

If $t_{2}=t_{3}$ for both beams (such as for round beams), then $\beta_{x_{+}}^{*}=\beta_{y_{+}}^{*}$ and $\beta_{x-}^{*}=\beta_{y-}^{*} ;$ we allc $;$ however, for the possibility that $\beta_{x+}^{*} \neq \beta_{x-}^{*}$ and we is ume nothing about the six rms beam sizes. Then we find that

$$
R_{x+}(0)=R_{y+}(0)=\rho+(1-\rho) \sqrt{\pi} t_{2} \exp \left(t_{2}^{2}\right) \operatorname{erfc}\left(t_{2}\right)
$$

where $\operatorname{erfc}(x)$ is the complementary error function. $R_{x-}(0)=R_{y-}(0)$ is obtained by exchanging $t_{1} \rightarrow t_{2}$.

For particles away from the bunch center, Eq. (4) implies that $\xi(z)=\xi(-z)$ for each of the four beam-beam parameters. This means that the particles at the ! ad and the tail of the bunch suffer the same beam-beam ti :e shift. This property follows from the assumed lattice sy imetry about the IP and the assumed lack of bunch disru "ion.

The aggravating factors saturate [2] to a limit wh:: $z \rightarrow$ $\infty$. In practice this limit applies to particles with $|z| \gg \beta^{*}$, where $\beta^{*}$ is here any of the four beta-functions at the IP; therefore this limit may or may not be sensibly reached in specific machine designs. Furthermore, this property follows from a first-order calculation; it may not hold in higher orders if $\xi(\infty)$ is large.

\section{The SLAC/LBL/LLNL B FACTORY}

For nominal parameters of the APIARY 6.3-D design for the proposed SLAC/LBL/LLNL B factory [1] we obtain $t_{x}=47.43$ and $t_{y}=1.897$, so that $R=0.945$. This implies that the luminosity is $5.5 \%$ smaller than the zero-bunchlength estimate.

We also obtain $R_{y+}(0)=1.093$, so that the vertical beam-beam parameter of the central positron is $9.3 \%$ larger than the nominal value. The other three aggravating factors are slightly smaller than unity [2]. For particles away from the center of the bunch, $R_{y+}$ grows almost linearly with $z$. Fig. 2 shows $\xi_{y+}$ as a function of the positron's longitudinal distance away from the center of the bunch. The remaining three aggravating factors deviate significantly from unity only for $z \gtrsim 10 \sigma_{3}$. Fig. 3 shows all four aggravating factors as a function of the particle's cistance away from the center of the bunch.

A qualitative estimate of the electromagnetic bunch disruption can be obtained from results for multiparticle simulations for single-pass, symmetric, bram collisions [7]. For flat bunches that are uniform in $x$ and gaussian in $y$ and $s$, one obtains, from Chen's empirical fit, that the disruption is $H_{D}=0.998$ with an estimated accuracy of $\pm 10 \%$, for nominal APIARY 6.3-D parameters $(A=0.53$ and $D=0.20$ ). Since $H_{D}$ takes into account both the geometrical and the electromagnetic disruption effects, we conclude, to this accuracy, that the geometrical reduction in luminosity is compensated by the pinching effect. This 


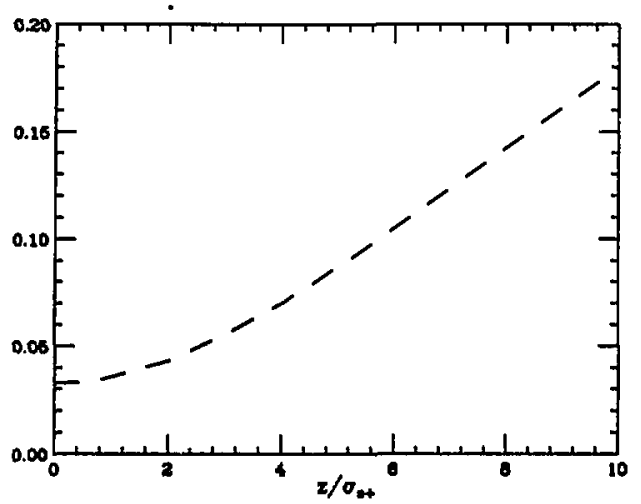

Figure 2: The vertical beam-beam parameter for the positron, $\xi_{y+}(z)$, for nominal APIARY 6.3-D parameters. The nominal value is $\xi_{0}=0.03$ for all four beam-beam parameters. The almost linear rise is explained in [2].

result is consistent with the multiparticle tracking simulation results for the SLAC/LBL/LLNL B factory when parasitic collisions are ignored [1]. One should keep in mind, however, that since Chen's results apply to singlepass collisions, a potentially important dependence on the tune of the machine may be missed in this interpretation.

\section{Conclusions}

As in the symmetric case, the luminosity reduction factor is a sensitive function of $\beta^{*} / \sigma_{\text {. }}$. Unlike the symmetric case, however, this factor depends explicitly on the transverse bunch sizes in addition to the bunch lengths and beta-functions.

A numerical application to the SLAC/LBL/LLNL B factory shows a $5.5 \%$ geometrical reduction of the luminosity and a $9.3 \%$ geometrical enhancement of the central positron's $\xi_{y}+$ relative to the nominal values.

Positrons with $x \simeq y \simeq 0$ at the head or tail of the bunch have higher $\xi_{y+}$ than the central positron due to the fact that they sample, on average, a higher $\beta_{y+}^{*}$ during the collision process $\left(\beta_{y_{+}}^{*}=1.5 \mathrm{~cm}\right.$ is the smallest of the four $\beta^{\circ}$ 's). Positrons with $|z|>6 \sigma$, have $\xi_{y+}>0.1$; this number can be made smaller, however, by a modest increase in $\beta_{y+}^{*}$ [2].

We estimate the electromagnetic pinching effect to be small, since it modifies the results of the geometrical calculations by $\sim \pm 10 \%$. It is probably beneficial for the luminosity, and it is probably detrimental for the beambeam parameters.

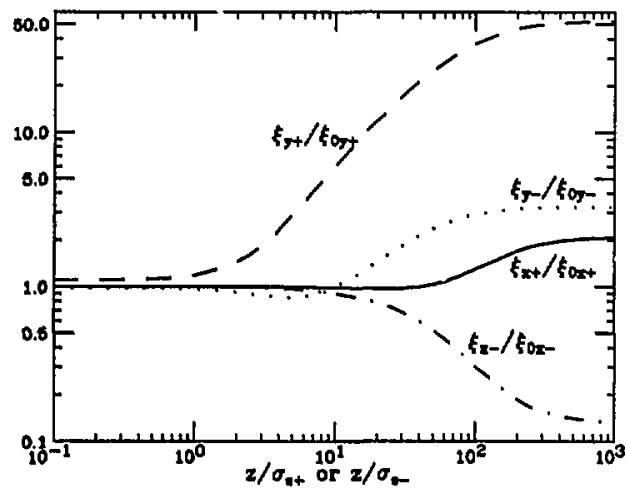

Figure 3: The aggravating factors for both beams for nominal APIARY 6.3-D parameters. The suturation property is seen at (unphysically) large values of $z\left(\sigma_{\mathrm{s}}=1 \mathrm{~cm}\right)$.

\section{ACKNOWLEDGMENTS}

I thank Pisin Chen, Yong-Ho Chil1, Bill Fawley, sinctrew Hutton, Jeff Tennyson and Mike Zisman for discussions.

\section{REFERENCES}

[1] LBL PUB-5303/SLAC-372/CALT-68-1715/UCRLID-106426/UC-IIRPA-91-01, February 1991; CLNS 91-1050; KEK 90-24, March 1991.

[2] M. A. Furman, ABC-21/ESG-161, April 1991.

[3] Y. II. Chin, AIP Conf. Proc. 214, 424 (1990); also Y. H. Chin, LBL-27665, 1989; M. A. Furman, ABC25/ESG-161, February 1991.

[4] S. Milton, CBN 89-1.

[5] P. M. Ivanov, I. A. Koop, E. A. Perevedentsev, Yu. M. Shatunov and I. B. Vasserman, 1989 ICFA Workshop, Novosibirsk.

[6] J. T. Seeman, Springer-Verlag Lecture Notes in Physics no. 247, Sardinia, 1985.

[7] P. Chen, SLAC-PUB-4822, December 1988, Snowmass 1988; P. Chen and K. Yokoya, Phys Rev. D 38, 987 (1988). 